\title{
Comparing 3T T1-Weighted Sequences in Identifying Hyperintense Punctate Lesions in Preterm Neonates
}

\author{
D. Tortora, V. Panara, P.A. Mattei, A. Tartaro, R. Salomone, S. Domizio, A.R. Cotroneo, and M. Caulo
}

\begin{abstract}
BACKGROUND AND PURPOSE: The loss of contrast on T1-weighted MR images at 3T may affect the detection of hyperintense punctate lesions indicative of periventricular leukomalacia in preterm neonates. The aim of the present study was to determine which $3 \mathrm{~T}$ T1weighted sequence identified the highest number of hyperintense punctate lesions and to explore the relationship between the number of hyperintense punctate lesions and clinical outcome.
\end{abstract}

MATERIALS AND METHODS: The presence of hyperintense punctate lesions was retrospectively evaluated in 200 consecutive preterm neonates on 4 axial T1-weighted sequences: 3-mm inversion recovery and spin-echo and 1- and 3-mm reformatted 3D-fast-field echo. Statistically significant differences in the number of hyperintense punctate lesions were evaluated by using a linear mixed-model analysis. Logistic regression analysis was used to assess the relation between the number of hyperintense punctate lesions and neuromotor outcome at 3 months.

RESULTS: Thirty-one neonates had at least 1 hyperintense punctate lesion indicative of periventricular leukomalacia in at least 1 of the 4 sequences. The 1-mm axial reformatted 3D-fast-field echo sequence identified the greatest number of hyperintense punctate lesions $(P<$ $.001)$. No statistically significant differences were found among the 3-mm T1-weighted sequences. The greater number of hyperintense punctate lesions detected by the 1-mm reformatted T1 3D-fast-field echo sequence in the central region of the brain was associated with a worse clinical outcome.

CONCLUSIONS: At 3T, the 1-mm axial reformatted T1 3D-fast-field echo sequence identified the greatest number of hyperintense punctate lesions in the central region of preterm neonate brains, and this number was associated with neuromotor outcome.

ABBREVIATIONS: FFE = fast-field echo; IR = inversion recovery; HPL = hyperintense punctate lesion; ICC = intraclass correlation coefficient; $\mathrm{PN}=$ preterm neonate; $\mathrm{PVL}=$ periventricular leukomalacia; $\mathrm{SE}=$ spin-echo

$\mathbf{P}$ eriventricular white matter injury is a major form of brain damage and the leading cause of chronic neurologic disability in survivors of preterm births. ${ }^{1,2}$ Periventricular white matter injury includes a spectrum of cerebral damage that ranges from focal necrotic lesions (periventricular leukomalacia [PVL]) to diffuse myelination disturbances (diffuse periventricular white matter injury). ${ }^{3-5}$ PVL is pathologically characterized by foci of

Received March 10, 2014; accepted after revision August 14.

From the Radiology Unit (D.T., V.P., A.R.C., M.C.), Ophthalmology Clinic (P.A.M.), and Neonatology and Neonatal Intensive Care Unit (R.S., S.D.), University Hospital of Chieti, Chieti, Italy; and ITAB-Institute of Advanced Biomedical Technologies (D.T. V.P., A.T., M.C.) and Department of Neuroscience and Imaging (A.T., A.R.C., M.C.), University "G. d'Annunzio" of Chieti, Chieti, Italy.

Preliminary results previously presented at: Annual Meeting of the European Congress of Radiology, March 7-11, 2013; Vienna, Austria.

Please address correspondence to Massimo Caulo, MD, PhD, ITAB-Institute of Advanced Biomedical Technologies, University “G. d'Annunzio," Via Dei Vestini 33, 66100 Chieti, Italy; e-mail: caulo@unich.it

http://dx.doi.org/10.3174/ajnr.A4144 coagulation necrosis in the periventricular white matter, which results in degeneration of cellular elements and, in particular, the premyelinating oligodendrocytes. The physiopathologic mechanism underlying PVL remains an important unresolved question. ${ }^{6}$

Classic MR imaging signs of PVL are hyperintense punctate lesions (HPLs) on T1-weighted sequences, which infrequently may also appear hypointense on T2-weighted images. ${ }^{7}$ More severe presentations of PVL are either cavitation (cystic PVL) or glial scars and may be accompanied by a conspicuous reduction in white matter volume and ventricular enlargement. ${ }^{5}$ The causes determining the shortening of T1 and T2 signals in punctate PVL have not been completely established. Some authors hypothesized that signal shortening was determined by the presence of focal hemorrhages. Other studies hypothesized that the characteristic hyperintense signal on T1-weighted sequences was due to the contemporary presence of hemosiderin deposition, lipids from myelin breakdown, and dystrophic calcifications. ${ }^{7}$ 
Table 1: MRI sequence parameters

\begin{tabular}{|c|c|c|c|c|c|c|c|c|}
\hline Sequence & Section Thickness (mm) & Intersection (mm) & TR (ms) & $\mathrm{TE}$ (ms) & $\mathrm{TI}(\mathrm{ms})$ & FA & SAR (W/Kg) & dB \\
\hline Axial TI SE & 3 & 0.5 & 663 & 11 & - & - & $<0.2$ & -6.5 \\
\hline Axial TI IR & 3 & 0.5 & 4938 & 15 & 400 & - & $<0.1$ & 7.2 \\
\hline T1 3D-FFE & 1 and 3 & 0 & 9.2 & 4.3 & - & $10^{\circ}$ & 0.0 & 5.4 \\
\hline Axial T2-TSE & 3 & 0.5 & 3000 & 80 & - & - & $<0.2$ & 6.5 \\
\hline Axial DWI & 4 & 1 & 2380 & 65 & - & - & $<0.1$ & 9.5 \\
\hline
\end{tabular}

Note:-SAR indicates specific absorption rate; FA, flip angle.

Preterm children with PVL present a high incidence of neurodevelopmental disabilities such as cerebral palsy; mental retardation; and psychologic, developmental, behavioral, and emotional disorders. ${ }^{8}$ Currently, an accurate method of identifying all preterm neonates (PNs) at risk of poor developmental outcome is not available, to our knowledge. Clinical risk factors, cranial sonography, and electroencephalography are used to study PNs around birth but are not optimal predictors of long-term outcome. ${ }^{9,10}$ MR imaging can provide prognostic information concerning neuropsychological development. For example, PNs with a greater number of HPLs at MR imaging subsequently presented a worse motor and neurocognitive outcome. More specifically, the presence of HPLs in white matter regions traversed by the motor pathway was shown to be a useful predictor of motor outcome. ${ }^{11,12}$ However, the use of MR imaging to screen PNs has been relatively limited because of the availability of the MR imaging systems, the complex management of neonates, and, above all, the low prognostic accuracy when no lesions are identified. ${ }^{13,14}$

To date, most MR imaging studies on PVL were performed by using MR imaging systems operating at $1.5 \mathrm{~T}$ or lower field strengths. Liauw et $\mathrm{al}^{15}$ concluded that the spin-echo (SE) T1weighted sequence scored best for detecting the presence of HPLs.

Recently, the benefits from the improved signal-to-noise ratio and higher spatial resolution of MR imaging systems operating at $3 \mathrm{~T}$ indicated the potential of this high magnetic field system for studying the neonatal brain, though the elongation of the T1 relaxation time at higher magnetic field strengths ${ }^{16}$ may bias the use of $3 \mathrm{~T}$ scanners in preterm neonates due to the potential reduction of the sensitivity of T1 images for identifying HPLs.

The aim of the present study was to determine which 3T T1weighted sequence identified the highest number of HPLs in PNs studied at term-corrected age and to explore the relationship between the number/location of HPLs and the clinical outcome.

\section{MATERIALS AND METHODS \\ Patients}

The institutional review board approved our retrospective study and waived informed consent. The brain MR imaging acquisitions of 200 consecutive PNs acquired from January 2010 to December 2012 within 5 days of term-corrected age as part of an ongoing screening of premature neonates (study approved by the Ethics Committee of “G. D'Annunzio" University of Chieti and Azienda Sanitaria Locale n.2 of Lanciano-Vasto-Chieti) were included in this study. Parents or legal guardians of the neonates always gave written informed consent to the MR imaging study.

\section{Neuromotor Outcome}

Neurologic examinations of the PNs were routinely performed at 3 months of corrected age by a pediatric neurologist who assigned the infant to 1 of the 3 following groups, according to the classification of development by the World Health Organization: 1) "normal" (normal neurologic examination findings); 2) "mildly abnormal" (mild hypertonia, hypotonia, and/or asymmetry); and 3) "definitely abnormal" (cerebral palsy). ${ }^{17}$ Results were obtained from patient records.

\section{MR Imaging}

MR imaging was performed by using a 3T whole-body system (Achieva 3.0T X-Series; Philips Healthcare, Best, the Netherlands) with a dedicated 8-channel head coil. Neonates were fed and sedated with 10-mg oral chloral hydrate per kilogram of body weight. During the scan, the neonates were laid in a supine position and swaddled in blankets. Molded foam was placed around the body of the neonate to minimize head movement. Ear protection was always used and consisted of commercially available neonatal earmuffs (MiniMuffs; Natus Medical, San Carlos, California) and adapted ear-canal plugs. Heart rate and oxygen saturation were monitored during the MR imaging study by an experienced intensive care neonatologist. All neonates underwent the same MR imaging protocol. Details of the MR imaging sequences are reported in Table 1.

\section{MR Image Analysis}

Phase 1. Two neuroradiologists, with 15 and 5 years of experience in neonatal neuroimaging, reviewed, in consensus, the MR imaging studies of the 200 neonates. They performed a preliminary image-quality assessment of the acquired sequences by using a 3 -point scale ( 0 , nondiagnostic images; 1 , diagnostic image with limitations; and 2, fully diagnostic). Assessed parameters included general visual image quality, image noise, and the presence of artifacts. Only MR imaging sequences with scores of at least 2 were included in subsequent analyses.

Phase 2. Four weeks later, the same 2 neuroradiologists independently reviewed the MR imaging studies selected in phase 1 . They focused on the presence of HPLs on the 4 T1-weighted sequences (3-mm axial SE, 3-mm axial inversion recovery [IR], and 3- and 1-mm axial reformatted 3D-fast-field echo [FFE]). An HPL was defined as a hyperintense punctate lesion on $\mathrm{T} 1$ with a diameter of $<5 \mathrm{~mm}$ that did not necessarily present a corresponding decreased signal intensity on T2-weighted images.

A standardized system of visual assessment was used. The 2 readers, blinded to neonate identity, clinical history, and gestational age at birth, used a workstation equipped with a professional DICOM viewer (OsiriX Imaging Software; http://www. osirix-viewer.com). They evaluated the T1-weighted sequences for the following characteristics: 1) number of HPLs, 2) location: anterior to the frontal horn of the lateral ventricles (anterior re- 
gion), posterior to the trigon (posterior region), or in the centrum semiovale and/or corona radiata (central region traversed by the motor pathway). ${ }^{5}$ The sequences of all neonates were randomly presented.

Phase 3. Four weeks later the same 2 neuroradiologists jointly reviewed all the MR imaging studies in which at least 1 HPL was detected during phase 2 . They were asked to reach a consensus on a global HPL evaluation (number and location). This consensus reading was considered the reference standard for statistical analysis.

\section{Statistical Analysis}

Statistical analysis was performed by using SPSS Statistics for Mac, Version 21.0 (IBM, Armonk, New York). The level of significance was set at $P<.05$.

Linear mixed-model analysis was performed to evaluate the presence of significant differences between the overall number of HPLs identified in each T1-weighted sequence and the number of HPLs identified in each location (anterior, posterior, and central/motor).

The presence of a relationship between the neuromotor outcome at 3 months and the number of HPLs was tested by using logistic regression with a backward stepwise conditional method.

Intraclass correlation coefficients (ICCs) were used to study the agreement between the 2 readers in visualizing the number of HPLs with each T1 sequence. An ICC of $<0.40$ indicated a poor agreement; 0.40-0.75, fair-to-good (moderate); and 0.76-1.00, excellent agreement. ${ }^{18}$ To analyze the performance of each MR imaging sequence in showing the presence of HPLs, we calculated the ICC between the individual assessments and the reference standard.

\section{RESULTS}

\section{Patients}

The MR imaging studies of 184/200 (92\%) PNs were scored as at least 2 at the preliminary image-quality analysis and therefore were included in the analysis. Thirty-one of 184 (16.8\%) PNs (12 females; average gestational age, $32.5 \pm 2.5$ weeks; range, $28-36$ weeks) presented with at least $1 \mathrm{HPL}$ on at least 1 T1-weighted sequence and were included in subsequent statistical analysis. In 4 of these neonates, T1 SE images were not obtained due to patient awakening. In 1 neonate, both SE and T1 IR images were not obtained.

\section{Neuromotor Outcome}

At 3 months, 20/31 (64.5\%) preterm infants presented with a mildly abnormal motor development (spasticity), 9/31 (29.0\%) presented with a normal motor development, none of the patients presented with cerebral palsy, and 2/31 (6.5\%) PNs were lost to follow-up.

\section{Number of HPLs}

In consensus reading, the $1-\mathrm{mm}$ reformatted $3 \mathrm{D}-\mathrm{FFE}$ sequence detected the greatest overall number of HPLs ( $n=386$ lesions); 3-mm reformatted 3D-FFE, T1 IR, and T1 SE sequences identified 218,152 , and 151 HPLs, respectively. In 7 PNs, the presence of
Table 2: HPLs identified in consensus reading

\begin{tabular}{|c|c|c|c|c|c|c|}
\hline \multirow[b]{2}{*}{ Patient } & \multirow[b]{2}{*}{ Sex } & \multirow[b]{2}{*}{ Outcome } & \multicolumn{4}{|c|}{ Overall } \\
\hline & & & $\begin{array}{l}\text { 3-mm } \\
\text { T1 FFE }\end{array}$ & $\begin{array}{l}\text { 1-mm } \\
\text { T1 FFE }\end{array}$ & $\begin{array}{c}\text { 3-mm } \\
\mathrm{TI} I \mathrm{R}\end{array}$ & $\begin{array}{c}3-\mathrm{mm} \\
\mathrm{TI} \mathrm{SE}\end{array}$ \\
\hline 1 & $\mathrm{~F}$ & 1 & 4 & 7 & 0 & NA \\
\hline 2 & $\mathrm{~F}$ & 0 & 3 & 7 & 0 & NA \\
\hline 3 & $\mathrm{~F}$ & 1 & 12 & 20 & 6 & NA \\
\hline 4 & $\mathrm{~F}$ & 0 & 3 & 8 & 6 & NA \\
\hline 5 & $M$ & 1 & 8 & 12 & 5 & 4 \\
\hline 6 & $F$ & 1 & 16 & 35 & 8 & 16 \\
\hline 7 & $M$ & 0 & 9 & 12 & 6 & 7 \\
\hline 8 & $M$ & 1 & 17 & 19 & 10 & 11 \\
\hline 9 & M & 1 & 12 & 25 & 9 & 9 \\
\hline 10 & $\mathrm{~F}$ & 1 & 9 & 11 & 11 & 10 \\
\hline 11 & $M$ & 0 & 3 & 5 & 2 & 4 \\
\hline 12 & $\mathrm{~F}$ & NA & 5 & 14 & 7 & 4 \\
\hline 13 & $M$ & 1 & 6 & 9 & 11 & 11 \\
\hline 14 & $M$ & 1 & 21 & 30 & 9 & 9 \\
\hline 15 & M & 1 & 15 & 29 & 17 & 15 \\
\hline 16 & $M$ & 1 & 6 & 13 & 8 & 14 \\
\hline 17 & $M$ & NA & 4 & 9 & NA & NA \\
\hline 18 & $\mathrm{~F}$ & 0 & 8 & 11 & 0 & 0 \\
\hline 19 & $M$ & 0 & 6 & 9 & 1 & 2 \\
\hline 20 & $\mathrm{~F}$ & 1 & 6 & 12 & 5 & 6 \\
\hline 21 & $M$ & 0 & 4 & 9 & 0 & 0 \\
\hline 22 & $M$ & 1 & 4 & 8 & 3 & 4 \\
\hline 23 & $M$ & 1 & 2 & 4 & 2 & 2 \\
\hline 24 & $\mathrm{~F}$ & 1 & 4 & 10 & 3 & 1 \\
\hline 25 & $M$ & 1 & 1 & 7 & 5 & 2 \\
\hline 26 & $M$ & 1 & 15 & 15 & 9 & 13 \\
\hline 27 & $M$ & 0 & 5 & 10 & 3 & 3 \\
\hline 28 & $M$ & 0 & 2 & 5 & 0 & 0 \\
\hline 29 & $M$ & 1 & 2 & 8 & 0 & 0 \\
\hline 30 & $F$ & 1 & 1 & 5 & 0 & 0 \\
\hline 31 & $\mathrm{~F}$ & 1 & 5 & 8 & 6 & 4 \\
\hline Total & & & 218 & 386 & 152 & 151 \\
\hline
\end{tabular}

Note:-NA indicates not available.

HPLs was identified exclusively on the reformatted 3D-FFE sequences and not on SE and IR. Results are summarized in Table 2.

The consensus reading of the location-related number of HPLs indicated that in the central/motor location, 1- and 3-mm reformatted 3D-FFE sequences detected 227 and 115 HPLs. The T1 IR and T1 SE sequences identified 67 and $71 \mathrm{HPLs}$, respectively. In the anterior location, 1- and 3-mm reformatted 3D-FFE sequences detected 26 and 18 HPLs. The IR and SE sequences identified 18 and 12 HPLs, respectively. In the posterior location, 1- and 3-mm reformatted 3DFFE sequences detected 133 and 85 HPLs. The IR and SE sequences identified 67 and 68 HPLs, respectively.

The linear mixed-model analysis showed a statistical difference among the overall number of lesions detected by the 4 T1weighted sequences $(P<.01)$. Comparison of the means indicated that the 1-mm reformatted T1 3D-FFE sequence identified a significantly greater overall number of HPLs than 3-mm reformatted T1 3D-FFE, SE, and IR sequences. No statistical differences were observed among the overall number of lesions detected by 3 -mm sequences $(P>.05)$ (Fig 1 ).

Linear mixed-model analysis showed a statistical difference among the number of HPLs detected by the 4 T1-weighted sequences in the central/motor location $(P<.01)$. Comparison of the means indicated that the $1-\mathrm{mm}$ reformatted T1 3D-FFE sequence identified a significantly greater number of HPLs than the SE and IR sequences $(P<.01)$, whereas no differences were ob- 


\section{LOCATIONS}

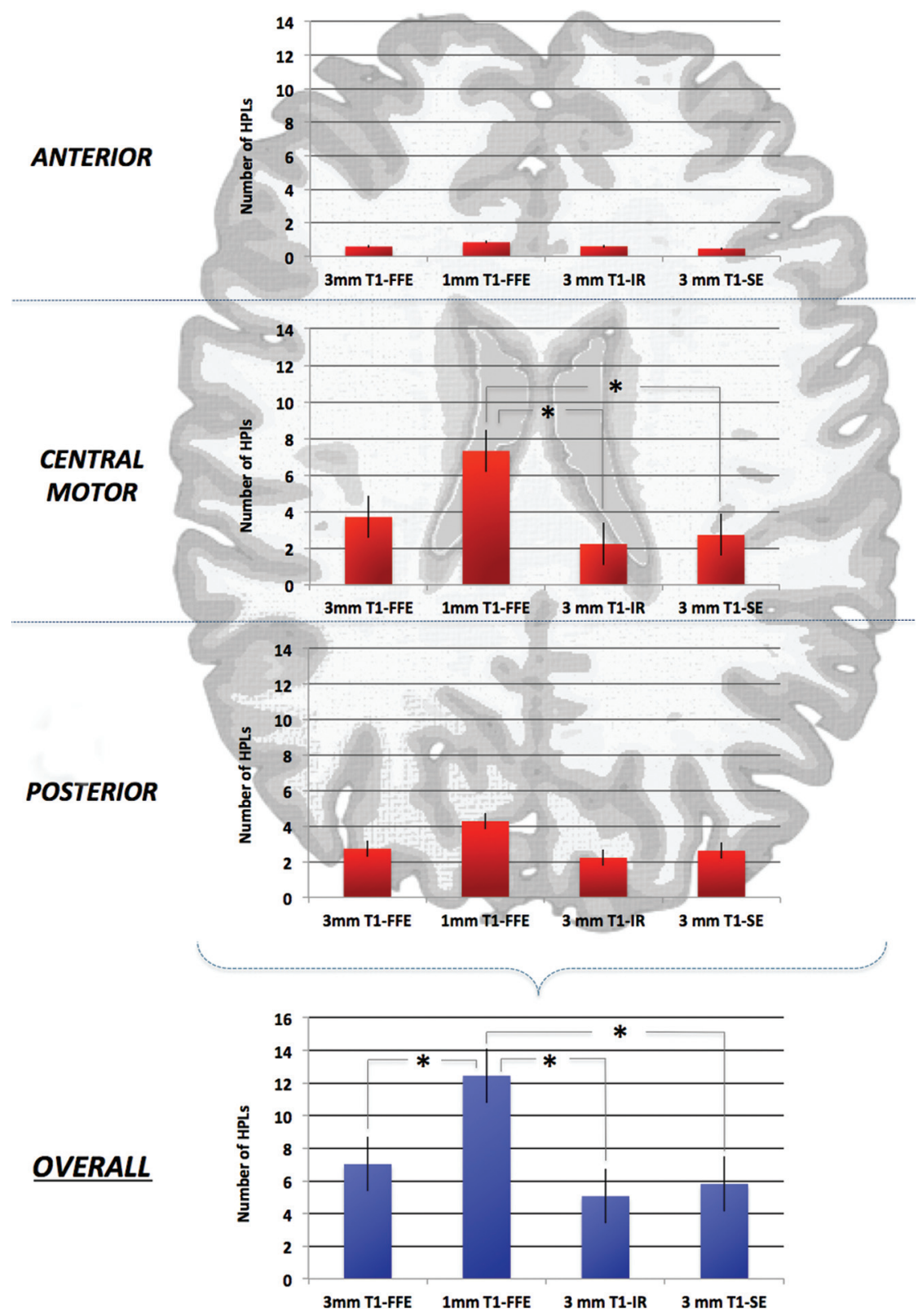

FIG 1. Schematic representation of a typical neonatal brain. Only the central (motor) location on the 1-mm reformatted T7 3D-FFE sequence scored a significantly greater number of HPLs compared with IR and SE sequences. The 1-mm axial reformatted TI 3D-FFE sequence identified a significant overall greater number of HPLs than other $3-\mathrm{mm}$ sequences $(P<.01)$. sequence was included in the primary logistic regression analysis, and 3-mm reformatted T1 3D-FFE, IR, and SE sequences were removed from the backward stepwise model. The number of HPLs detected by the 1-mm T1 3D-FFE sequence was an independent predictive factor for neuromotor outcome (odds ratio $=1.690 ; 95 \% \mathrm{CI}, 1.017-2.807 ; P=$ $.04)$.

\section{Interreader Agreement Analysis}

A moderate-to-excellent interreader agreement was observed for all the sequences. The greatest agreement was reached by using the 1-mm axial reformatted T1 3D-FFE sequence (ICC = 0.986). Results of the interreader agreement analysis are summarized in Table 3.

The ICCs of each T1-weighted sequence in relation to the reference standard indicated that the 1-mm axial reformatted T1 3D-FFE sequence yielded the best results (excellent agreement) for both readers. The agreement between the 3-mm T1-weighted sequences and the criterion standard yielded a higher ICC for the 3D-FFE (excellent agreement) than for the SE and IR sequences (moderate agreement) for both readers (Table 3 ).

\section{DISCUSSION}

Despite the recent availability of an everincreasing number of $3 \mathrm{~T}$ scanners, specific indications for the identification of HPLs in PNs are still lacking. Therefore, in this 3T study, we evaluated the contribution of different T1-weighted sequences for the identification and localization of HPLs in a group of PNs. The results indicated that axial reformatted $\mathrm{T} 1$ 3D-FFE scored better than T1 IR and T1 SE sequences for detecting HPLs, though only the 1-mm sequence differed significantly from the others. These findings can be explained by the combination of thin- served with the $3-\mathrm{mm}$ reformatted $3 \mathrm{D}$-FFE sequence $(P>.05)$. Statistically significant differences were not observed among the number of HPLs identified by the 4 T1-weighted sequences in anterior and posterior locations (Fig 1).

\section{Relationship between Neuromotor Outcome and the Number of HPLs}

To test the clinical implication of the only significant difference in the detection score of the HPLs by the 4 different T1-weighted sequences, we tested the relationship between the number of HPLs in the central region and the neuromotor outcome at 3 months. The number of HPLs detected by each T1-weighted ner section thickness and the intrinsically higher sensitivity of T1-weighted FFE sequences for hyperintense lesions compared with SE. Wintersperger et $\mathrm{al}^{19}$ demonstrated that T1 3D-FFE sequences showed a higher signal-to-noise ratio compared with T1 SE sequences at both $1.5 \mathrm{~T}$ and $3 \mathrm{~T}$. Edelman et $\mathrm{al}^{20}$ recently reinforced this finding by demonstrating that at $3 \mathrm{~T}$ the $\mathrm{T} 13 \mathrm{D}$-FFE presented a significantly higher quality compared with the 2D T1-SE images, providing a higher contrast-to-noise ratio for discriminating gray and white matter structures, reducing artifacts, and improving imaging efficiency with reformatted images in orthogonal orientations. In the present study, the same reasons can be given to explain the difference in scoring HPLs observed between the T1 3D-FFE and T1 IR se- 
quences - that is, the T1 IR sequence had the same spin magnetization refocusing of the T1 SE. This aspect was also confirmed by the lack of a significant difference in HPL detection between the T1 SE and T1 IR sequences.

In addition to the intrinsically higher SNR of FFE compared with SE sequences, the section thickness and the possibility of obtaining an isotropic voxel are important in increasing the sensitivity of FFE sequences.

The T1 3D-FFE sequence with a section thickness of $1 \mathrm{~mm}$ scored better in identifying HPLs than the other 3-mm T1weighted sequences $(P<.01)$. In particular, the difference between 1 - and 3-mm axial reformatted T1 3D-FFE sequences $(P<$ $.01)$ demonstrated that decreasing the section thickness can significantly increase the detection rate of punctate lesions. This outcome is because the use of thinner sections allowed the separation of contiguous lesions that were seen as confluent with thicker sections (ie, cluster-organized HPLs could be identified as multiple lesions at $1 \mathrm{~mm}$, while they appeared as a single lesion at $3 \mathrm{~mm}$ ) (Fig 2).

The importance of using a 3D-FFE sequences was reinforced by the overall greater number of appropriately identified PVLs by using both the 1- and 3-mm reformatted images compared with $\mathrm{SE}$ and IR sequences.

Table 3: Interreader agreement and pulse sequence performances $^{\mathrm{a}}$

\begin{tabular}{cccc} 
Pulse & $\begin{array}{c}\text { Agreement of } \\
\text { Reader 1 and } \\
\text { Reference } \\
\text { Standard }\end{array}$ & $\begin{array}{c}\text { Agreement of } \\
\text { Reader 2 and } \\
\text { Reference } \\
\text { Standard }\end{array}$ & $\begin{array}{c}\text { Interreader } \\
\text { Agreement }\end{array}$ \\
\hline 3-mm T1 FFE & 0.893 & 0.822 & 0.844 \\
1-mm T1 FFE & 0.988 & 0.992 & 0.986 \\
3-mm T1 IR & 0.776 & 0.631 & 0.653 \\
3-mm T1 SE & 0.793 & 0.797 & 0.820 \\
\hline
\end{tabular}

${ }^{a}$ Data are intraclass correlation coefficients.

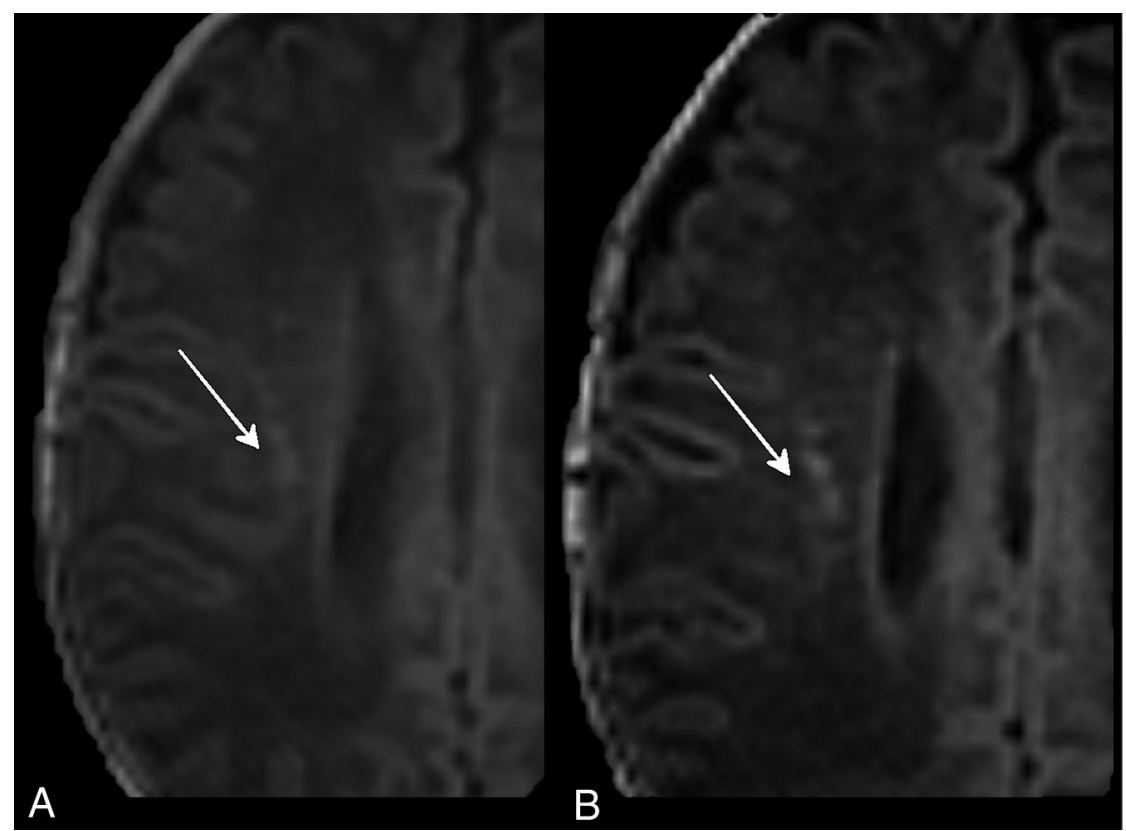

FIG 2. Three-millimeter $(A)$ and 1-mm $(B)$ axial reformatted T1 3D-FFE sequences of PNs affected by PVL. The thinner sections $(B)$ separated lesions that were seen as confluent with the thicker sections $(A)$.
As a second step, we classified HPLs according to their location (posterior, central, and anterior). ${ }^{12}$ We observed that the $1-\mathrm{mm}$ reformatted 3D-FFE sequence scored a statistically significantly greater number of HPLs compared with the IR and SE sequences only in the central (motor) location. This finding may be attributed to the intrinsically higher contrast-to-noise ratio of T1 3DFFE sequences that discriminated HPLs from the relatively higher T1 signal intensity of the myelinated cortical spinal tract of neonates. On the other hand, the absence of statistically significant differences in the anterior and posterior locations among the different T1-weighted sequences may be attributed to the lower T1signal intensity of the unmyelinated white matter, which increased the contrast with HPLs.

On the basis of the findings reported by Nanba et al, ${ }^{12}$ subsequent analysis focused on the central (motor) location. We evaluated the relationship between the number of HPLs detected by each sequence and the neuromotor outcome at 3 months. The greater number of HPLs detected by the $1-\mathrm{mm}$ reformatted T1 $3 \mathrm{D}-\mathrm{FFE}$ sequence positively correlated to a worse clinical outcome, thus suggesting that this sequence should be used in routine clinical use for PNs.

The ICCs of interreader agreement in the detection of HPLs were moderate to excellent for all the T1-weighted sequences except for IR, which had the lowest score performance $(\mathrm{ICC}=0.63$ ) and interobserver agreement $(\mathrm{ICC}=0.66)$. These results seem to contradict a previous $3 \mathrm{~T}$ study by Stehling et al, ${ }^{21}$ which evaluated differences in contrast-to-noise ratio, artifacts, gray-white differentiation, image noise, and overall diagnostic quality among FFE, IR, and SE sequences. They demonstrated a higher contrast-tonoise ratio and a better gray-white matter differentiation with a higher overall diagnostic quality of both FFE and IR compared with SE sequences. We hypothesize that this discrepancy was related to the lower negative influence of the inhomogeneous myelination of the white matter of PNs on the FFE sequences. ${ }^{20,21}$

The highest interreader agreement was obtained with the 1-mm reformatted T1 3D-FFE sequence. This result could be related to the higher HPL conspicuity on this sequence, due to the higher intrinsic SNR and thinner section thickness. In addition, the higher interreader agreement obtained with T1 3D-FFE compared with T1 IR and T1 SE sequences demonstrated that the detection of HPLs by using the T1 3D-FFE sequence was less influenced by reader experience.

Another important advantage of 3DFFE sequences is their intrinsic lower specific absorption rate compared with SE. The specific absorption rate level increases with field strength and is higher in T1 SE sequences due to the refocusing pulses and in T1 IR sequences because of their intense radiofrequency pulse. ${ }^{21}$ The possibility of using lower specific absorption rate levels is particularly important in neonates due to their immature thermoregulatory abilities. 
The main limitation of this study was the lack of a direct comparison between 1.5 and $3 \mathrm{~T}$ sequences. For ethical reasons, we decided not to repeat the MR imaging studies at different magnetic field intensities.

An additional limit of this study was its retrospective design. The only clinical outcome that was consistently available from patient records for the study group was the neuromotor one at 3 months. In addition, our study population lacked cases with the most unfavorable neuromotor outcome (cerebral palsy). These points should be addressed in future studies. In addition, 4 neonates had incomplete MR imaging studies because they did not undergo axial T1-SE and one did not undergo T1 IR.

All nonisometric sequences were acquired on the axial plane; thus, a comparison with sagittal and coronal reformatted 3D-FFE sequences was not possible. ${ }^{12}$ The patient population imposed a rigid limit to the duration of the acquisition, thus excluding the possibility of also acquiring all sequences in the coronal plane. Nonetheless, an evaluation of the effect of the acquisition plane was beyond the scope of this study.

\section{CONCLUSIONS}

This study demonstrated that with $3 \mathrm{~T}$ scanners, the 1-mm axial reformatted T13D-FFE sequence identified the greatest number of HPLs in the central region of PN brains, and this number was predictive of neuromotor outcome at 3 months.

\section{REFERENCES}

1. Volpe JJ. Neurology of the Newborn. Philadelphia: W.B. Saunders; 2000

2. Ferriero DM. Neontal brain injury. N Engl J Med 2004;351:1985-95

3. Volpe JJ. Neurobiology of periventricular leukomalacia in the premature infant. Pediatr Res 2001;50:553-62

4. Rezaie P, Dean A. Periventricular leukomalacia, inflammation and white matter lesions within the developing nervous system. Neuropathology 2002;22:106-32

5. Back SA, Riddle A, McClure MM. Maturation-dependent vulnerability of perinatal white matter in premature birth. Stroke 2007; 38(2 suppl):724-30

6. Distefano G, Praticò AD. Actualities on molecular pathogenesis and repairing processes of cerebral damage in perinatal hypoxic-ischemic encephalopathy. Ital J Pediatr 2010;16:36:63
7. Cornette LG, Tanner SF, Ramenghi LA, et al. Magnetic resonance imaging of the infant brain: anatomical characteristics and clinical significance of punctate lesions. Arch Dis Child Fetal Neonatal Ed 2002;86:F171-77

8. Olsén P, Paakkii E, Vainionpaa L, et al. Magnetic resonance imaging of periventricular leukomalacia and its clinical correlation in children. Ann Neurol 1997;41:754-61

9. Ment LR, Hirtz D, Huppi PS. Imaging biomarkers of outcome in the developing preterm brain. Lancet Neurol 2009;8:1042-55

10. Leijser LM, de Bruïne FT, van der Grond J, et al. Is sequential cranial ultrasound reliable for detection of white matter injury in very preterm infants? Neuroradiology 2010;52:397-406

11. Hintz SR, O'Shea M. Neuroimaging and neurodevelopmental outcomes in preterm infants. Semin Perinatol 2008;32:11-19

12. Nanba Y, Matsui K, Aida N, et al. Magnetic resonance imaging regional $\mathrm{T} 1$ abnormalities at term accurately predict motor outcome in preterm infants. Pediatrics 2007;120:e10-19

13. Bax M, Tydeman C, Flodmark O. Clinical and MRI correlates of cerebral palsy: the European Cerebral Palsy Study. JAMA 2006; 296:1602-08

14. Back SA, Miller SP. Brain injury in premature neonates: a primary cerebral dysmaturation disorder? Ann Neurol 2014;75:469-86

15. Liauw L, van der Grond J, van den Berg-Huysmans AA, et al. Hypoxic-ischemic encephalopathy: diagnostic value of conventional MR imaging pulse sequences in term-born neonates. Radiology 2008;247:204-12

16. Williams LA, Gelman N, Picot PA, et al. Neonatal brain: regional variability of in vivo $\mathrm{MR}$ imaging relaxation rates at $3.0 \mathrm{~T}$-initial experience. Radiology 2005;235:595-603

17. Rosenbaum P, Stewart D. The World Health Organization international classification of functioning, disability and health: a model to guide clinical thinking, practice and research in the field of cerebral palsy. Semin Pediatr Neurol 2004;11:5-10

18. Fleiss JL. Statistical Methods for Rates and Proportion. 2nd ed. New York: John Wiley; 1981

19. Wintersperger BJ, Runge VM, Biswas J, et al. Brain tumor enhancement in MR imaging at 3 Tesla: comparison of SNR and CNR gain using TSE and GRE techniques. Invest Radiol 2007;42:558-63

20. Edelman RR, Dunkle E, Koktzoglou I, et al. Rapid whole-brain magnetic resonance imaging with isotropic resolution at 3 Tesla. Invest Radiol 2009;44:54-59

21. Stehling C, Niederstadt T, Krämer S. Comparison of a T1weighted inversion-recovery-, gradient-echo- and spin-echo sequence for imaging of the brain at 3.0 Tesla [in German]. Rofo 2005; $177: 536-42$ 